\title{
Phase Retrieval, Symmetrization Rule and Transport-of-Intensity Equation in Application to Induction Mapping of Magnetic Materials
}

\author{
V.V. Volkov and Y. Zhu \\ Brookhaven National Laboratory, Materials Science Department, Upton, NY 11973
}

Recent progress in the field of noninterferometric phase retrieval brings the ordinary Fresnel microscopy to a new quantitative level, suitable for recovering both the amplitude and phase of the object, based on image intensity measurements of the object [1,2]. We show that this is sufficient for in-plane component mapping of magnetic induction [2] for small magnetic elements with known geometry ranging from micro- to few nanometers size.

In present paper we re-examine some conservation principles used for the transport-of-intensity (TIE) equation derived by Teaque [3] for application to phase retrieval in light and X-ray optics. In particular, we prove that the intensity conservation law [3] should be replaced in general case with the energy-flow conservation law [2]. This law describes the amplitude-phase balance of the partially coherent beam on its propagation along the optical path, valid both for light and electron optics. This substitution has at least two important fundamental consequences.

First, we formulate in a good approximation the new boundary condition [2], suitable for unique phase recovery from TIE up to some arbitrary constant

$$
\left[\mathbf{n} \cdot \nabla_{\perp} \varphi(L)\right]=\partial \varphi(L) / \partial \mathbf{n}=0,
$$

Here $L=\partial D$ is the image $D(x, y)$ perimeter and $\partial \varphi / \partial \mathbf{n}$ is the phase gradient in the direction of outward normal. Notice that Neumann boundary condition (1) is sufficient to fulfil the intensity conservation law in the paraxial wave approximation as suggested by Teaque [3]. This condition is of great practical importance since (a) it can be applied for a wide class of objects, (b) it is well suited for fast phase retrieval via Fourier transform (FT) methods and (c) it does not require the use of an aperture while recording experimental images. The practical application of this condition is realized through the "symmetrization rule" [2] of experimental images as described in Figs.1-2. Notice that phase information recovered by this rule via FT is free from edge spoiling/aliasing effects in the entire area of experimental image.

The second consequence is important for phase retrieval from ordinary Fresnel images of magnetic materials obtained by Lorentz microscopy. In particular, a new so-called magnetic transport-ofintensity (MTIE) equation [4] is derived under the assumption of uniform thickness of magnetic film. This new equation makes the basis for noninterferometric magnetic induction $\boldsymbol{B}(\mathrm{x}, \mathrm{y})$ mapping in TEM based on the intensity $I\left(\mathrm{x}, \mathrm{y}, \mathrm{z}_{\mathrm{o}} \pm \delta \mathrm{z}\right)$ measurements versus image defocus. In principle, the problem is reduced to the solution of nonlinear elliptic differential equation (MTIE) of second orler in a manner similar to TIE-phase solution known for partially coherent light optics [5]. Fig. 3 shows the MTIE solution for $\mathrm{B}(\mathrm{x}, \mathrm{y})$ mapping in $\mathrm{Nd}_{2} \mathrm{Fe}_{14} \mathrm{~B}$ magnet after the first iteration of phase retrieval algorithm. The derivation of MTIE equation and its solution will be presented as well.[6]

References:

1. S. Bajt et al. Ultramicroscopy 83 (2000) 67; D. Paganin, K.A. Nugent, PRL, 80 (1998) 2586.

2. V.V. Volkov, Y. Zhu, M. De Graef, Micron, /JMIC580/ (2002), in-press.

3. M. R. Teague, J. Opt. Soc.Am. 72, 1199 (1982); J. Opt. Soc. Am.73 (1983) 1434.

4. V.V. Volkov and Y. Zhu, J. Appl. Phys. (2002) submitted.

5. T.E. Gureev, A. Roberts, K.A. Nugent. J. Opt. Soc. Am. A12 (1995) 1942.

6. Work supported by US DOE, DE-AC02-98CH10886. 


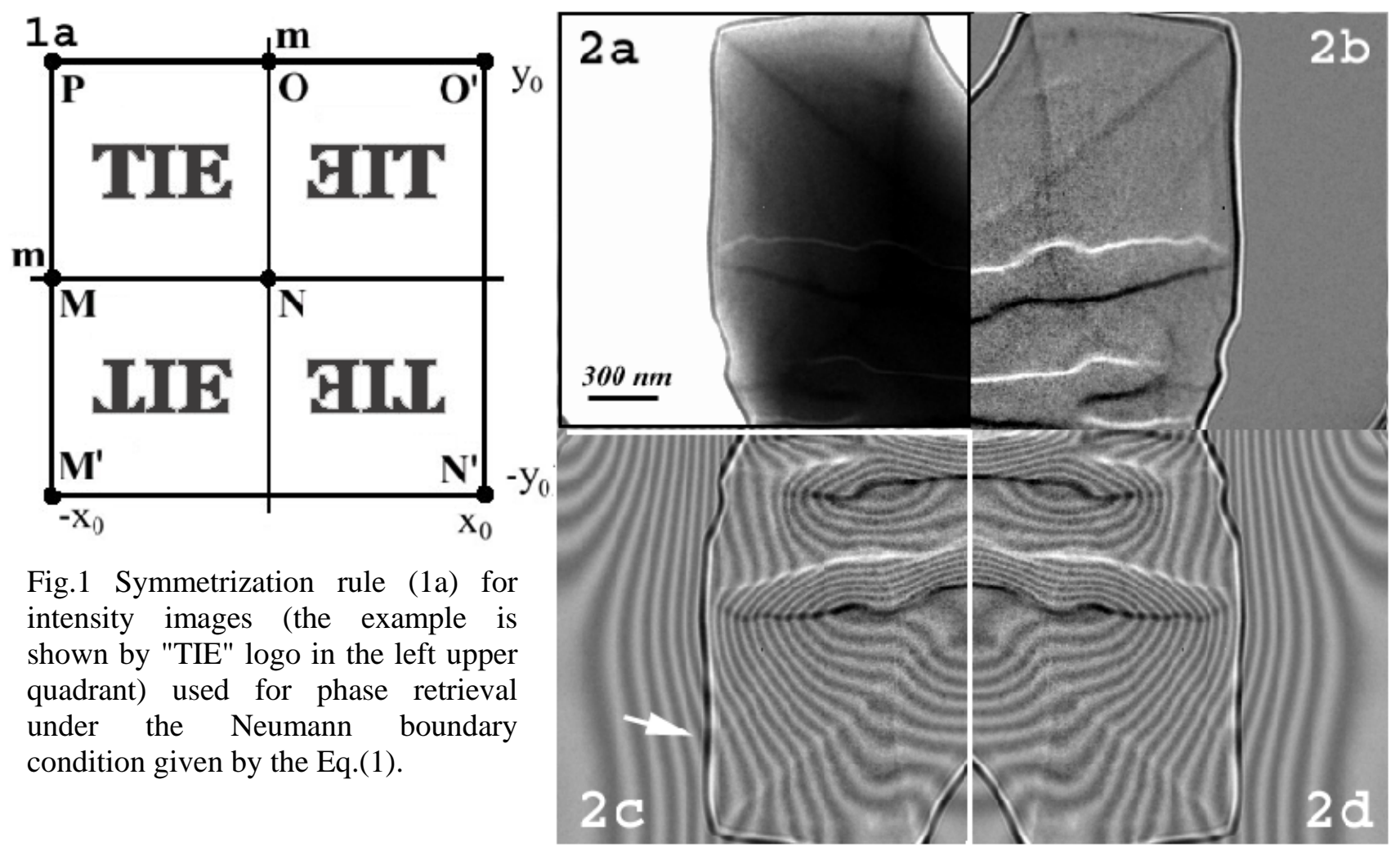

Fig.2 Example of TEM induction mapping using defocused Fresnel images (2a) of magnetic domains in $\mathrm{Nd}_{2} \mathrm{Fe}_{14} \mathrm{~B}$ hard magnet. Experimental (2a) and phase-contrast (2b) images obtained by Fresnel imaging. MTIE-phase reconstruction process (2c) shown after the first iteration of retrieval algorithm displayed by the flux line contours corresponding to magnetization of sample imaged in (2a). Non-spoiled FT-solution in (2c) is obtained by introduction of mirrored (virtual) image (2d) as required by the symmetrization rule. Notice a good convergence of MTIE-algorithm in spite of difficult imaging conditions, since many details of magnetic structure can be revealed after the first iteration.
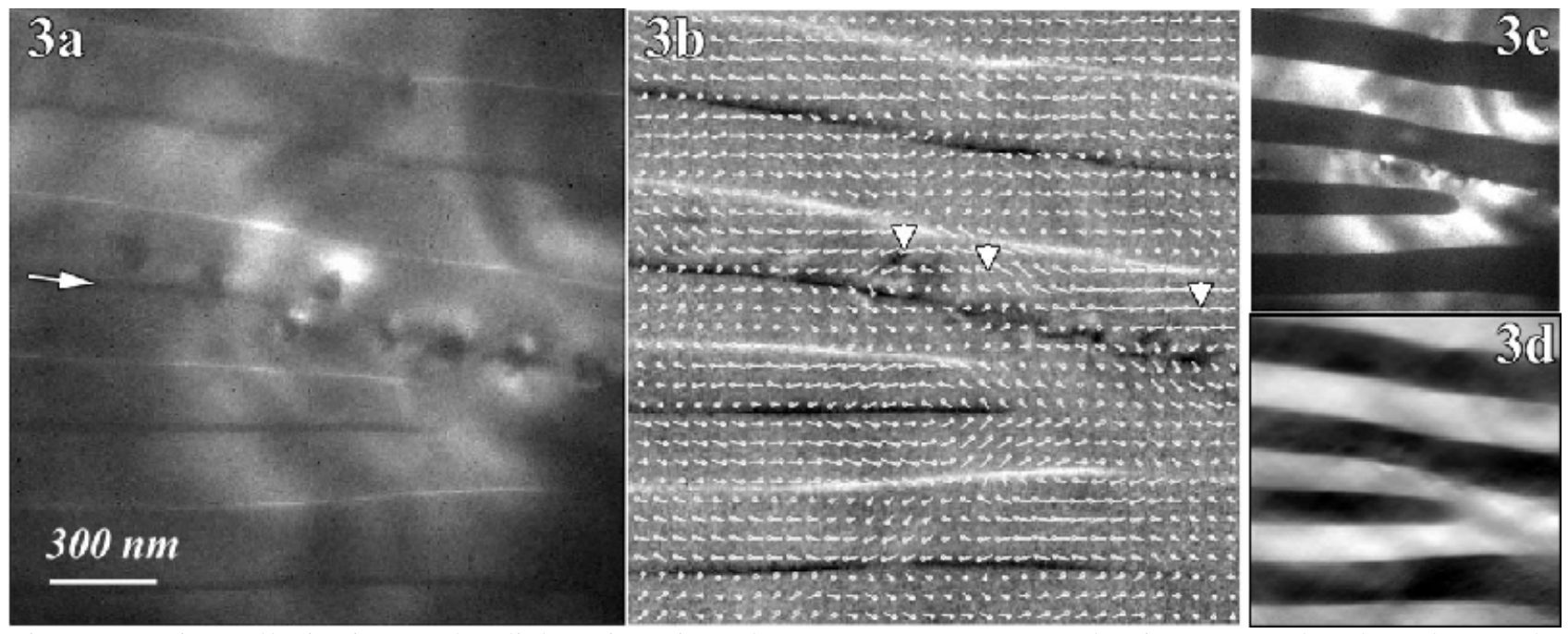

Fig.3 Domain wall pinning at the dislocations in $\mathrm{Nd}_{2} \mathrm{Fe}_{14} \mathrm{~B}$ magnet (3a). Induction map (3b) plotted over the phase contrast image. Experimental Foucault image (3c) compared with calculated one (3d) derived from the induction map (3b) after removal of background intensity. Notice weak magnetization turbulence (arrowed) at the dislocation core positions. 